\title{
CUMULATIVE INDEX
}

Volumes $1-20$

\section{HOW TO USE THE INDEX}

The Cumulative Index consists of four sections:

1. Author Index. Entries include regular articles, special articles, and technical articles and notes published in this Journal, arranged alphabetically by author. For a particular first author, entries are arranged chronologically, without regard to multiple authorship (on the assumption that the user is more likely to be familiar with the chronology of a particular author's papers than with the details of multiple authorship). Cross-references to co-authored papers for which the particular author was not first author are grouped together at the end of the chronological listing for that author.

2. Subject Index. Entries are based on all articles within this Journal, including special articles and technical articles and notes. Entries for the major kinds of reinforcement schedules (DRL, FI, FR, VI, and VR) have been classified under two major headings, the first covering studies in which the schedule is treated as an independent variable, and the second covering studies in which the schedule is used as a baseline. For experimental subjects (e.g., rat), indexing is limited primarily to those studies concerned with a species-specific problem or with an interspecies comparison. In some cases, entries for compound terms are listed under the noun rather than the adjective (e.g., articles dealing with behavioral contrast may be found under contrast, behavioral).

3. Tables of Contents. In each of the above indices, articles in this Journal are cited by volume number and page: $(2,23)$ represents Volume 2 , page 23 . The author(s) and title for such a citation may be located without leaving the Cumulative Index by consulting the Contents Index, in which articles are arranged by volume and page number.

4. Book Reviews. For the reader's convenience, titles have been repeated in this separate listing. 